\title{
Fat mass- and obesity-associated genotype, dietary intakes and anthropometric measures in European adults: the Food4Me study
}

\author{
Katherine M. Livingstone ${ }^{1} \dagger$, Carlos Celis-Morales ${ }^{1} \dagger$, Santiago Navas-Carretero ${ }^{2,3}$,
} Rodrigo San-Cristobal ${ }^{2,3}$, Hannah Forster ${ }^{4}$, Clare B. O'Donovan ${ }^{4}$, Clara Woolhead ${ }^{4}$, Cyril F. M. Marsaux ${ }^{5}$, Anna L. Macready ${ }^{6}$, Rosalind Fallaize ${ }^{6}$, Silvia Kolossa ${ }^{7}$, Lydia Tsirigoti ${ }^{8}$, Christina P. Lambrinou ${ }^{8}$, George Moschonis $^{8}$, Magdalena Godlewska ${ }^{9}$, Agnieszka Surwiłło ${ }^{9}$, Christian A. Drevon ${ }^{10}$, Yannis Manios ${ }^{8}$, Iwona Traczyk $^{9}$, Eileen R. Gibney ${ }^{4}$, Lorraine Brennan ${ }^{4}$, Marianne C. Walsh ${ }^{4}$, Julie A. Lovegrove ${ }^{6}$, J. Alfredo Martinez ${ }^{2,3}$, Wim H. M. Saris ${ }^{5}$, Hannelore Daniel ${ }^{7}$, Mike Gibney ${ }^{4}$ and John C. Mathers ${ }^{1 *}$ on behalf of the Food4Me study

${ }^{1}$ Human Nutrition Research Centre, Institute of Cellular Medicine, Newcastle University, Newcastle Upon Tyne NE1 7RU, UK

${ }^{2}$ Center for Nutrition Research, University of Navarra, 31008 Pamplona, Spain

${ }^{3}$ CIBER Fisiopatología Obesidad y Nutrición (CIBERobn), Instituto de Salud Carlos III, Madrid, Spain

${ }^{4}$ UCD Institute of Food and Health, University College Dublin, Belfield, Dublin 4, Republic of Ireland

${ }^{5}$ Department of Human Biology, NUTRIM School of Nutrition and Translational Research in Metabolism, Maastricht University Medical Centre, Maastricht 6229 HX, The Netherlands

${ }^{6}$ Hugh Sinclair Unit of Human Nutrition and Institute for Cardiovascular and Metabolic Research, University of Reading, Reading RGG 6AP, UK

${ }^{7}$ ZIEL Research Center of Nutrition and Food Sciences, Biochemistry Unit, Technische Universität München, 85435 Freising, Germany

${ }^{8}$ Department of Nutrition and Dietetics, Harokopio University, Kallithea 17671, Greece

${ }^{9}$ National Food E Nutrition Institute (IZZ), Warsaw 02903, Poland

${ }^{10}$ Department of Nutrition, Institute of Basic Medical Sciences, Faculty of Medicine, University of Oslo, O372 Oslo, Norway

(Final revision received 9 July 2015 - Submitted 22 September 2015 - Accepted 28 October 2015-First published online 1 December 2015)

\section{Abstract}

The interplay between the fat mass- and obesity-associated (FTO) gene variants and diet has been implicated in the development of obesity. The aim of the present analysis was to investigate associations between FTO genotype, dietary intakes and anthropometrics among European adults. Participants in the Food4Me randomised controlled trial were genotyped for FTO genotype (rs9939609) and their dietary intakes, and diet quality scores (Healthy Eating Index and PREDIMED-based Mediterranean diet score) were estimated from FFQ. Relationships between FTO genotype, diet and anthropometrics (weight, waist circumference (WC) and BMI) were evaluated at baseline. European adults with the FTO risk genotype had greater WC ( $A A v$. TT: $+1.4 \mathrm{~cm} ; P=0.003)$ and BMI $\left(+0.9 \mathrm{~kg} / \mathrm{m}^{2} ; P=0.001\right)$ than individuals with no risk alleles. Subjects with the lowest fried food consumption and two copies of the FTO risk variant had on average $1.4 \mathrm{~kg} / \mathrm{m}^{2}$ greater BMI $\left(P_{\text {trend }}=0.028\right)$ and $3 \cdot 1 \mathrm{~cm}$ greater WC $\left(P_{\text {trend }}=0 \cdot 045\right)$ compared with individuals with no copies of the risk allele and with the lowest fried food consumption. However, there was no evidence of interactions between FTO genotype and dietary intakes on BMI and WC, and thus further research is required to confirm or refute these findings.

Key words: Fat mass- and obesity-associated gene: BMI: Fried foods: Dietary intakes

Over the past 30 years, the prevalence of obesity has increased markedly, with $17 \%$ of European adults ${ }^{(1)}$ and $9 \%$ of adults globally now being obese ${ }^{(2)}$. Obesity is a multifactorial condition that is influenced by the complex interplay between diet, physical activity (PA) and genetics ${ }^{(3,4)}$. Recent genome-wide association studies in nearly 400000 individuals have identified
SNP in genes, including the fat mass- and obesity-associated gene (FTO), which are strongly associated with the development of obesity $^{(5-7)}$. A study of 38759 individuals revealed that those homozygous for the FTO (rs9939609) risk allele weighed on average $3 \mathrm{~kg}$ more and had 1.7 -fold increased odds of being obese compared with those homozygous for the lower-risk allele ${ }^{(5)}$.

Abbreviations: EI, total energy intake; FTO, fat mass- and obesity-associated gene; MD, PREDIMED-based Mediterranean diet score; PA, physical activity; RCT, randomised controlled trial; WC, waist circumference.

* Corresponding author: Professor J. C. Mathers, fax +44 191208 1101, email john.mathers@newcastle.ac.uk

$\dagger$ These authors contributed equally to this work. 
The association between FTO and obesity has been attributed to higher energy intake ${ }^{(8,9)}$, although findings are equivocal ${ }^{(10)}$. Furthermore, obese individuals may consume higher amounts of energy-dense foods such as fried foods ${ }^{(11)}$. The obesogenic influence of FTO may be exacerbated by higher energy and fat intakes $^{(12,13)}$, although there is little information on links between FTO genotype and total dietary intake, or dietary patterns ${ }^{(14)}$. Interactions between obesity-susceptibility genes, intakes of fried foods and sugar-sweetened beverages and measures of adiposity have been reported among US adults ${ }^{(15,16)}$. Qi et $a l .{ }^{(15)}$ found that BMI increased by 1.1 (SE 0.2), 1.6 (SE 0.3 ) and 2.2 (SE 0.6$) \mathrm{kg} / \mathrm{m}^{2}$ per ten obesity-related risk alleles with increasing frequency of fried food consumption (less than once, one to three times and four or more times a week, respectively; $P_{\text {for interaction }}<0.001$ ). Furthermore, Qi et $a l^{(16)}$ reported an interaction between sugar-sweetened beverage intake and genetic predisposition to obesity ( $P_{\text {for interaction }}<0.001$ ). It is also evident that healthier eating patterns - for example, the PREDIMED-based Mediterranean diet (MD) score - may modulate the effect of FTO genotype on $\operatorname{adiposity}^{(17)}$. However, few studies have investigated relationships between FTO genotype, dietary intakes and adiposity in European adults.

The present study investigated the associations of FTO genotype and BMI and waist circumference (WC) with dietary intakes and potential interactions between FTO genotype, dietary intake and adiposity (BMI and WC) in adults participating in the panEuropean Food4Me randomised controlled trial (RCT) ${ }^{(18)}$.

\section{Methods}

\section{Study population}

The Food4Me proof of principle study was a 6-month, pan-European, RCT including 1607 adults ${ }^{(18,19)}$. Participants were recruited between August 2012 and August 2013 across seven European recruitment sites: University College Dublin (Ireland); Maastricht University (The Netherlands); University of Navarra (Spain); Harokopio University (Greece); University of Reading (UK); National Food and Nutrition Institute (Poland); and Technical University of Munich (Germany).

\section{Dietary intakes}

Habitual dietary intake was quantified using an online FFQ and food habits questionnaire, developed and validated for this study $^{(20,21)}$. The Food4Me FFQ included 157 food items consumed frequently in each of the seven recruitment countries. Intakes of foods and nutrients were computed in real time using a food composition database based on McCance and Widdowson's The Composition of Foods ${ }^{(22)}$. A 'fried foods' category was created by summing the following foods: chips, spring rolls, fried processed chicken or poultry, fried fish in batter and fish fingers/fish cakes. Additional information on fried food consumption was obtained from a questionnaire on dietary habits ${ }^{(20)}$, which asked how often participants consumed fried food in the past month, with the following options: 'never', '1-3 times/month', 'once a week', '2-4 times/week', '5-6 times/ week' or 'once a day'. Responses to this question were aggregated into three categories: low $=$ 'never' and ' $1-3$ times/ month'; medium = 'once a week' and '2-4 times/week'; and high = '5-6 times/week' and 'once a day'. Participants responded to questions on dietary habits including salt use (i.e. how often do you add salt while cooking? and how often do you add salt at the table?) and fat consumption (i.e. what do you do with visible fat on meat?). Both questions relating to salt were scored on a five-point scale ranging from 'never' to 'always', which was aggregated to a three-point scale (low = 'never' and 'rarely'; medium = 'sometimes'; high = 'usually' and 'always'). For fat consumption, respondents could select whether they ate most, some or as little as possible of visible fat on meat or that they did not eat meat, and these responses were aggregated to produce three groups: high = 'ate most or some of the fat', medium $=$ 'ate as little as possible' or low $=$ 'did not eat meat'. Categories were also created for 'sweets and snacks' and 'sugarsweetened beverages'. 'Sweets and snacks' included sweet biscuits, cakes, flapjacks, muesli bars, buns, muffins, pastries, waffles, pancakes, crêpes, fruit pies, tarts, crumbles, sponge and milk puddings, ice cream, sorbets and jellies, chocolate and chocolate snack bars, sweets, sugar added to tea/coffee/cereal and crisps. 'Sugar-sweetened beverages' were restricted to fizzy soft drinks and did not include low-energy content or diet options. A Healthy Eating Index (HEI) was estimated according to the consumption of total and whole fruits, total vegetables, greens and beans, whole grains, dairy products, protein and fatty acids ${ }^{(23)}$. The MD score was estimated based on an adaptation of the PREDIMED fourteen-point criteria. In brief, participants scored 1 if they met one of the following criteria and 0 if they did not: higher intake of olive oil than other culinary fat and of white meat than red meat, high intake of fruits (including natural fruit juice) and vegetables, legumes, nuts, fish, wine and sofrito and a limited intake of red and processed meats, fats and spreads, soft drinks and commercial bakery goods, sweets and pastries ${ }^{(24)}$. Scores were summed and ranged between 0 and $14^{(25)}$. Details of the MD scoring system are provided in online Supplementary Table S1.

\section{Assessment of anthropometric and lifestyle measures}

Body weight $(\mathrm{kg})$, height $(\mathrm{m})$ and WC $(\mathrm{cm})$ were self-measured and self-reported. Participants were provided with information sheets and online video instructions in their own language on how to complete the measurements. BMI $\left(\mathrm{kg} / \mathrm{m}^{2}\right)$ was calculated using measures of body weight and height. Self-reported measurements were validated in a sub-sample of the participants across seven European countries and showed a high degree of reliability ${ }^{(26)}$. Physical activity level (PAL) and time spent sedentary $(\mathrm{min} / \mathrm{d})$ were estimated from triaxial accelerometers (TracmorD; Philips Consumer Lifestyle). Participants self-reported their current smoking status.

\section{Genotyping}

Participants collected buccal cell samples at baseline using Isohelix SK-1 DNA buccal swabs and Isohelix dried capsules and posted the samples to each recruiting centre for shipment to LCG Genomics. LCG Genomics extracted DNA and genotyped specific loci using $\mathrm{KASP}^{\mathrm{TM}}$ genotyping assays to provide 
bi-allelic scoring of FTO SNP - rs9939609 and rs1121980. These two SNP showed a high linkage disequilibrium ( $\left.r^{2} 0.96\right)$, and therefore results for rs1121980 are not reported. No significant deviation from the Hardy-Weinberg equilibrium was observed for rs9939609 $(0.51 ; P=0.48)$.

\section{Ethical approval and participant consent}

This study was conducted according to the guidelines laid down in the 1964 Declaration of Helsinki, and all procedures involving human subjects were approved by the Research Ethics Committees at each University or Research Centre delivering the intervention. All participants who expressed an interest in the study were asked to sign online consent forms at two stages in the screening process. These consent forms were automatically directed to the local study investigators to be counter-signed and archived $^{(18)}$. The Food4Me trial was registered as a RCT (NCT01530139) at Clinicaltrials.gov.

\section{Statistical analysis}

Data were analysed using Stata (version 13; StataCorp LP). Only baseline data were used for the present analyses. Results from descriptive analyses are presented as means and standard deviations for continuous variables or as percentages for categorical variables. Multinomial logistic regression was used to test for significant differences across categorical variables and multiple linear regression was used for continuous variables. Multiple linear regression analyses were used to test for associations $\left(P_{\text {trend }}\right)$ between anthropometric measures (BMI and WC) and FTO genotype, stratified according to tertiles of dietary intakes or dietary scores, with the exception of sugar-sweetened beverages, which was a dichotomous variable due to high numbers of non-consumers ( $n$ 899). Interactions between categories of dietary intakes and FTO genotype on $\mathrm{BMI}$ and WC were investigated by including an interaction term in the model. Analyses were adjusted for age, sex, country, PAL and smoking (smokers, non-smokers). Results were deemed significant at $P<0 \cdot 05$. Sensitivity analyses were used to test the effects of specific foods within the fried food category (chips, pizza and spring rolls; fried processed chicken and poultry; and fried fish in batter and fish products). Sensitivity analyses also excluded participants who reported energy intake lower than BMR $\times 1 \cdot 1^{(27)}$, where BMR was calculated using Oxford equations $^{(28)}$, and energy intakes $>18828 \mathrm{~kJ} / \mathrm{d}(>4500 \mathrm{kcal} / \mathrm{d})^{(29)}$. Adjustment for total energy intake (EI) was included in the sensitivity analyses when investigating the relationship between foods, FTO genotype and interactions with BMI and WC. In addition, continuous variables for dietary intakes and anthropometrics were used to test for interactions between dietary intakes and FTO genotype.

\section{Results}

\section{Participant characteristics}

Of the 1607 individuals randomised into the Food4Me RCT, data at baseline on FTO genotype, anthropometry and dietary intake were available for 1277 participants. As summarised in Table 1, $30 \%$ of individuals were overweight and $16 \%$ were obese. In addition, $22 \%$ of males and $22 \%$ of females had WC above healthy limits ( $>102$ and $>88 \mathrm{~cm}$, respectively). Each additional copy of FTO risk allele was associated with an increase in weight, WC and BMI ( $P_{\text {trend }}=0.005,0.003$ and 0.001, respectively). Furthermore, the percentage of participants who were overweight or obese was higher in carriers of the FTO risk allele (TT: 29 v. AA: $33 \% ; P=0.036$ and 13 v. 18.4\%; $P=0.019$, respectively) than non-carriers. There were no significant differences in sex distribution, age, PA, smoking prevalence and EI:BMR ratio between FTO genotypes (Table 1).

\section{Dietary intake and FTO genotype}

No significant differences in total energy and macronutrient intakes were detected between FTO genotypes (Table 2). However, individuals with two risk alleles for FTO consumed more high-fat dairy products $(P=0.001)$ and fewer crisps $(P=0.043)$ than individuals with no FTO risk alleles. No other significant differences were observed (data not shown). Individuals with no copies of the FTO risk allele added salt less frequently while cooking $(P=0.032)$ than those with two copies of the risk allele. MD and HEI scores did not differ between FTO genotypes. The relationships between dietary intake and BMI are presented in the online Supplementary Tables 2 and 3.

\section{Dietary intakes, FTO genotype and anthropometrics}

On the basis of FFQ responses, individuals with the lowest fried food consumption (first tertile) and two copies of the FTO risk variant had on average $1.4 \mathrm{~kg} / \mathrm{m}^{2}$ greater BMI $\left(P_{\text {trend }}=0.028\right)$ and $3.1 \mathrm{~cm}$ greater WC $\left(P_{\text {trend }}=0.045\right)$ compared with individuals with no copies of the risk allele with the lowest fried food consumption. Similarly, individuals with medium fried food consumption (second tertile) and two copies of the FTO risk variant had on average $1.2 \mathrm{~kg} / \mathrm{m}^{2}$ greater BMI $\left(P_{\text {trend }}=0.036\right)$ compared with individuals with no copies of the risk allele with the lowest fried food consumption. No significant relationships were identified in individuals with the highest fried food consumption. WC did not differ between genotypes in medium or highest fried food consumers and no significant interactions were observed (Fig. 1). These results were consistent when fried food consumption was estimated from the food habits questionnaire: participants who rarely consumed fried foods and had two copies of the risk allele had $1.3 \mathrm{~kg} / \mathrm{m}^{2}$ higher BMI compared with those with no risk alleles $\left(P_{\text {trend }}=0.008\right)$. Similarly, participants who frequently consumed fried foods and had two copies of the risk allele had $5.2 \mathrm{~kg} / \mathrm{m}^{2}$ higher BMI compared with those with no risk alleles $\left(P_{\text {trend }}=0 \cdot 027\right)$. No significant interactions were observed. BMI was higher in individuals with two copies of the FTO risk genotype and lowest sugar-sweetened beverage consumption (lowest consumption; $A A v$. TT: $\left.+1.4 \mathrm{~kg} / \mathrm{m}^{2} ; P_{\text {trend }}=0.003\right)$ and highest sweet and snack consumption (highest consumption; $A A v$. TT: $\left.+1.7 \mathrm{~kg} / \mathrm{m}^{2} ; P_{\text {trend }}=0.009\right)$ compared with individuals with no copies of the FTO risk genotype. BMI was higher in individuals with two copies of the FTO risk genotype and moderate and 
Table 1. Demographic characteristics of participants by fat mass- and obesity-associated (FTO) risk allele (Mean values and standard deviations; percentages)

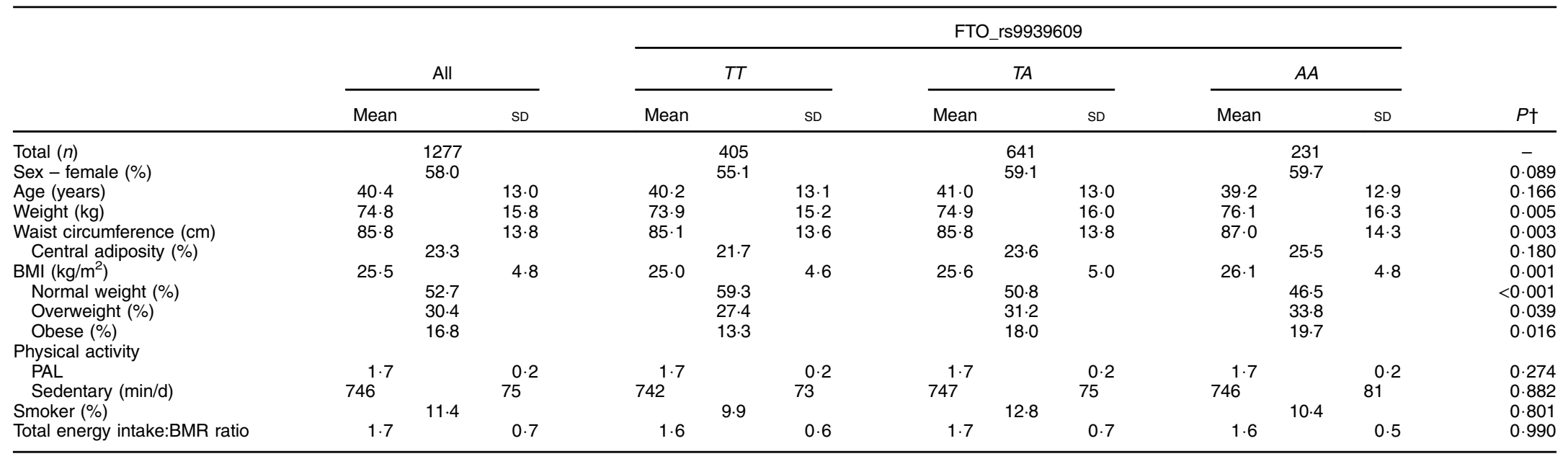

PAL, physical activity level (ratio between total energy expenditure:BMR)

* Central adiposity was defined as waist circumference $>88 \mathrm{~cm}$ in women and $>102 \mathrm{~cm}$ in men; normal: BMl 18.5-24.9 kg/m²; overweight: BMI 25-29.9 kg/m²; obese: BMl >30 kg/m².

† Multinomial logistic regression and multiple linear regression were used to test for significant differences across categorical and continuous variables, respectively; $P$ values were adjusted for age, sex, country and smoking habits. Analyses were also adjusted for BMI with the exception of weight, waist circumference and BMI.

Table 2. Dietary intakes of participants by fat mass- and obesity-associated (FTO) risk allele

(Mean values and standard deviations)

\begin{tabular}{|c|c|c|c|c|c|c|c|}
\hline & \multicolumn{6}{|c|}{ FTO_rs9939609 } & \multirow[b]{3}{*}{$P^{*}$} \\
\hline & \multicolumn{2}{|c|}{$T T$} & \multicolumn{2}{|c|}{$T A$} & \multicolumn{2}{|c|}{$A A$} & \\
\hline & Mean & SD & Mean & SD & Mean & SD & \\
\hline Total $(n)$ & \multicolumn{2}{|c|}{463} & \multicolumn{2}{|c|}{721} & \multicolumn{2}{|c|}{260} & \\
\hline Macronutrient intake & & & & & & & \\
\hline Total energy intake $(\mathrm{kJ})$ & 10594 & 3975 & 10868 & 4963 & 10565 & 3699 & 0.927 \\
\hline Fat (\% energy) & $36 \cdot 1$ & $6 \cdot 0$ & $35 \cdot 7$ & $5 \cdot 9$ & $36 \cdot 3$ & $5 \cdot 6$ & 0.595 \\
\hline SFA (\% energy) & $14 \cdot 2$ & $2 \cdot 9$ & $14 \cdot 0$ & $3 \cdot 3$ & 14.4 & $3 \cdot 0$ & 0.923 \\
\hline Trans-fat (\% energy) & 0.5 & 0.2 & 0.5 & 0.2 & 0.5 & 0.2 & 0.760 \\
\hline MUFA (\% energy) & 13.8 & $3 \cdot 2$ & 13.6 & 3.0 & 13.9 & $3 \cdot 3$ & 0.450 \\
\hline PUFA (\% energy) & $5 \cdot 8$ & 1.5 & $5 \cdot 8$ & 1.5 & $5 \cdot 7$ & $1 \cdot 3$ & 0.753 \\
\hline$n-3$ Fatty acids (\% energy) & 0.7 & 0.3 & 0.7 & 0.3 & 0.7 & 0.2 & 0.246 \\
\hline Carbohydrates (\% energy) & $45 \cdot 9$ & $8 \cdot 0$ & $46 \cdot 3$ & 7.5 & $45 \cdot 4$ & $7 \cdot 2$ & 0.959 \\
\hline Sugars (\% energy) & $21 \cdot 1$ & $6 \cdot 0$ & $21 \cdot 2$ & $6 \cdot 1$ & $20 \cdot 5$ & $5 \cdot 3$ & 0.471 \\
\hline Protein (\% energy) & $16 \cdot 9$ & $3 \cdot 6$ & $17 \cdot 1$ & $3 \cdot 8$ & $17 \cdot 3$ & 3.8 & 0.339 \\
\hline Alcohol (\% energy) & 3.5 & $3 \cdot 7$ & $3 \cdot 2$ & $3 \cdot 8$ & $3 \cdot 3$ & $3 \cdot 5$ & 0.587 \\
\hline Salt $(g / d)$ & $7 \cdot 3$ & $3 \cdot 3$ & 7.5 & 4.0 & $7 \cdot 3$ & $3 \cdot 3$ & 0.758 \\
\hline \multicolumn{8}{|c|}{ Contribution from sweets and snacks } \\
\hline Total energy & $15 \cdot 3$ & $9 \cdot 3$ & $14 \cdot 9$ & $10 \cdot 0$ & $15 \cdot 5$ & 9.7 & 0.802 \\
\hline$\%$ Energy from fat & 18.5 & 11.6 & $17 \cdot 7$ & 11.4 & $18 \cdot 3$ & 11.8 & 0.583 \\
\hline$\%$ Energy from SFA & $20 \cdot 9$ & $13 \cdot 5$ & $19 \cdot 7$ & $13 \cdot 2$ & $20 \cdot 7$ & $13 \cdot 6$ & 0.554 \\
\hline$\%$ Energy from sugars & $24 \cdot 7$ & $15 \cdot 1$ & $23 \cdot 9$ & $15 \cdot 5$ & $25 \cdot 5$ & $15 \cdot 7$ & 0.944 \\
\hline
\end{tabular}

* Multiple linear regressions were used to test for significant differences across genotypes. Analyses were adjusted for age, sex, physical activity, BMI, country and smoking status. 

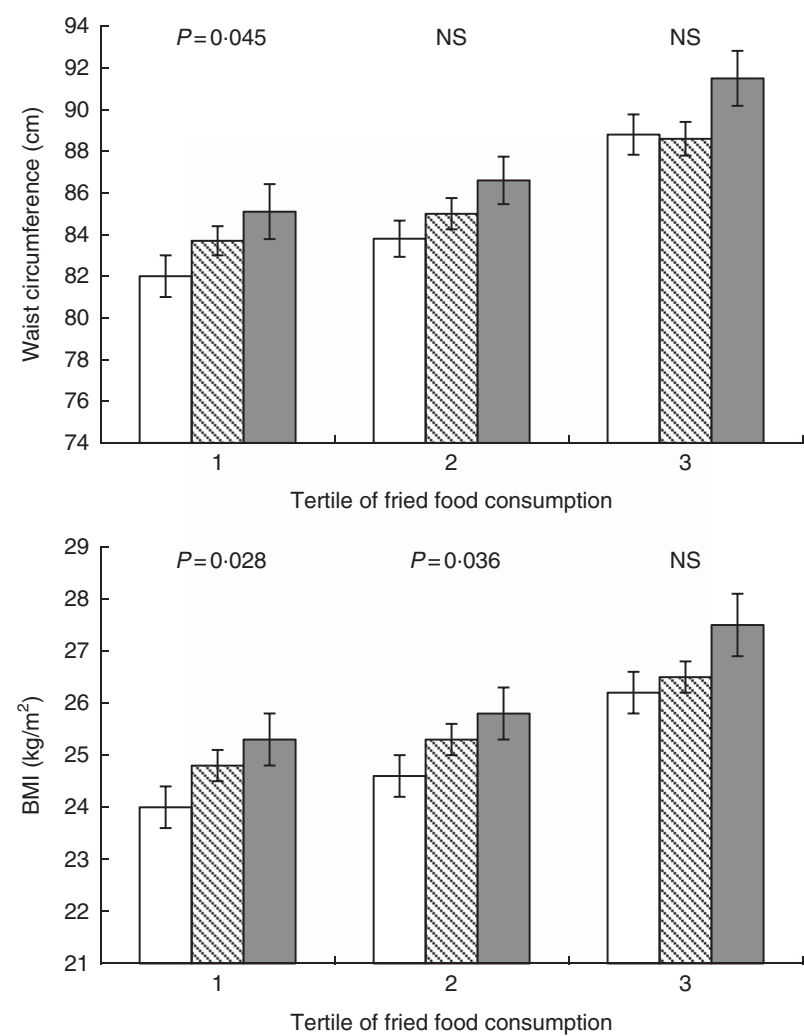

Fig 1. Anthropometric measures by tertile of fried food intake and fat massand obesity-associated risk allele. Values represent least squares means, with their standard errors adjusted for age, sex, country, physical activity and smoking status; tertile fried food: (1) $0-12.4 \mathrm{~g} / \mathrm{d}$; (2) $12.5-31.2 \mathrm{~g} / \mathrm{d}$, (3) 31.3-671 g/d. $\square, T T$; $\mathbb{N}, T A ; ~ \square, A$.

highest percentage energy intake from fat (second tertile: $+1.3 \mathrm{~kg} / \mathrm{m}^{2} ; P_{\text {trend }}=0.043$ and third tertile: $+1.8 \mathrm{~kg} / \mathrm{m}^{2} ; P_{\text {trend }}=$ $0 \cdot 004$, respectively) and lowest and highest percentage energy intake from sugar (first tertile: $+1.3 \mathrm{~kg} / \mathrm{m}^{2} ; P_{\text {trend }}=0.032$ and third tertile: $+1.9 \mathrm{~kg} / \mathrm{m}^{2} ; P_{\text {trend }}=0.004$, respectively) compared with individuals with no copies of the FTO risk genotype (Fig. 2). BMI was higher in individuals with two copies of the FTO risk genotype and low and high MD scores (first tertile: $+1.5 \mathrm{~kg} / \mathrm{m}^{2} ; P_{\text {trend }}=0.032$ and third tertile: $+1.8 \mathrm{~kg} / \mathrm{m}^{2} ; P_{\text {trend }}=$ 0.007 ) and low HEI score (first tertile: $+2.0 \mathrm{~kg} / \mathrm{m}^{2} ; P_{\text {trend }}=$ 0.004), compared with individuals with no copies of the FTO risk genotype (Fig. 2). With the exception of sugar-sweetened beverages $\left(P_{\text {interaction }}=0.049\right)$, no significant interactions between FTO genotype and dietary intakes on measures of adiposity were observed.

There were no significant trends in fried food consumption or percentage energy intake from total fat across FTO alleles when stratified by BMI category. Moreover, interactions between fried food consumption or percentage energy from total fat and FTO genotype on BMI were not significant (online Supplementary Fig. S1).

\section{Sensitivity analyses}

No significant interactions between specific sub-groups of fried foods and FTO genotype on anthropometric outcomes were observed. In addition, there were no significant interactions between FTO genotype and dietary intakes on BMI or WC when dietary intakes were included as continuous variables. Exclusion of energy misreporters did not change the pattern of the results. Adjustment for EI when investigating the relationship of food intakes with FTO genotype, as well as any interactions with BMI or WC, did not change the pattern of results (data not shown).

\section{Discussion \\ Main findings}

Our main finding is that individuals with the FTO risk genotype and the highest intakes of sugar, fat and sweet and snacks had the highest BMI. Furthermore, subjects with the lowest fried food consumption and two copies of the FTO risk variant had on average $1.4 \mathrm{~kg} / \mathrm{m}^{2}$ greater BMI $\left(P_{\text {trend }}=0.028\right)$ and $3.1 \mathrm{~cm}$ greater WC $\left(P_{\text {trend }}=0.045\right)$ compared with individuals with no copies of the risk allele and with the lowest fried food consumption. However, with the exception of sugar-sweetened beverages, we did not detect any significant interactions between dietary intakes and FTO genotype on BMI or WC. This is the first time that these relationships between genotype, diet and adiposity have been investigated in a pan-European population, and the link between 'unhealthy' dietary intakes and higher BMI in subjects with the risk alleles for FTO genotype warrants further investigation. Thus, further studies are required to confirm or to refute these observations.

\section{Comparison with other studies}

Our findings support the link between obesity and fried food consumption $^{(11,30)}$. A recent study in a US cohort reported that individuals with high genetic risk of obesity and high fried food consumption had $2 \cdot 4 \mathrm{~kg} / \mathrm{m}^{2}$ higher BMI than individuals with low genetic risk and low fried food intake $\left(P_{\text {for interaction }}=\right.$ $0 \cdot 005)^{(15)}$ These findings were replicated in two further cohorts with similar results ${ }^{(15)}$. Although the diet-gene interaction was not significant, when only the highest fried food consumers were considered, we demonstrated that European adults at higher genetic risk of obesity had $1.3 \mathrm{~kg} / \mathrm{m}^{2}$ higher BMI than individuals without the risk allele. Furthermore, with the exception of fried foods and sugar-sweetened beverages, we observed that BMI was significantly higher in the 'unhealthiest' tertile of dietary intake or dietary score. This suggests that the presence of the FTO risk allele may only increase BMI in individuals with the poorest diets.

Although we identified a significant interaction between sugar-sweetened beverages and genetic predisposition to obesity, this was the only significant interaction and may be a chance finding ${ }^{(16)}$. Importantly, our cohort of European adults was smaller (1280) compared with the Nurse's Health Study (9623), Health Professionals Follow-up Study (6379) and the Women's Genome Health Study (21 421), which may have impacted on our ability to detect significant interactions ${ }^{(15,16)}$. Furthermore, we did not detect any significant differences in macronutrient intakes or levels of PA between FTO genotypes, despite finding significant differences in BMI, WC and weight. Nonetheless, as investigated in a recent systematic review and 

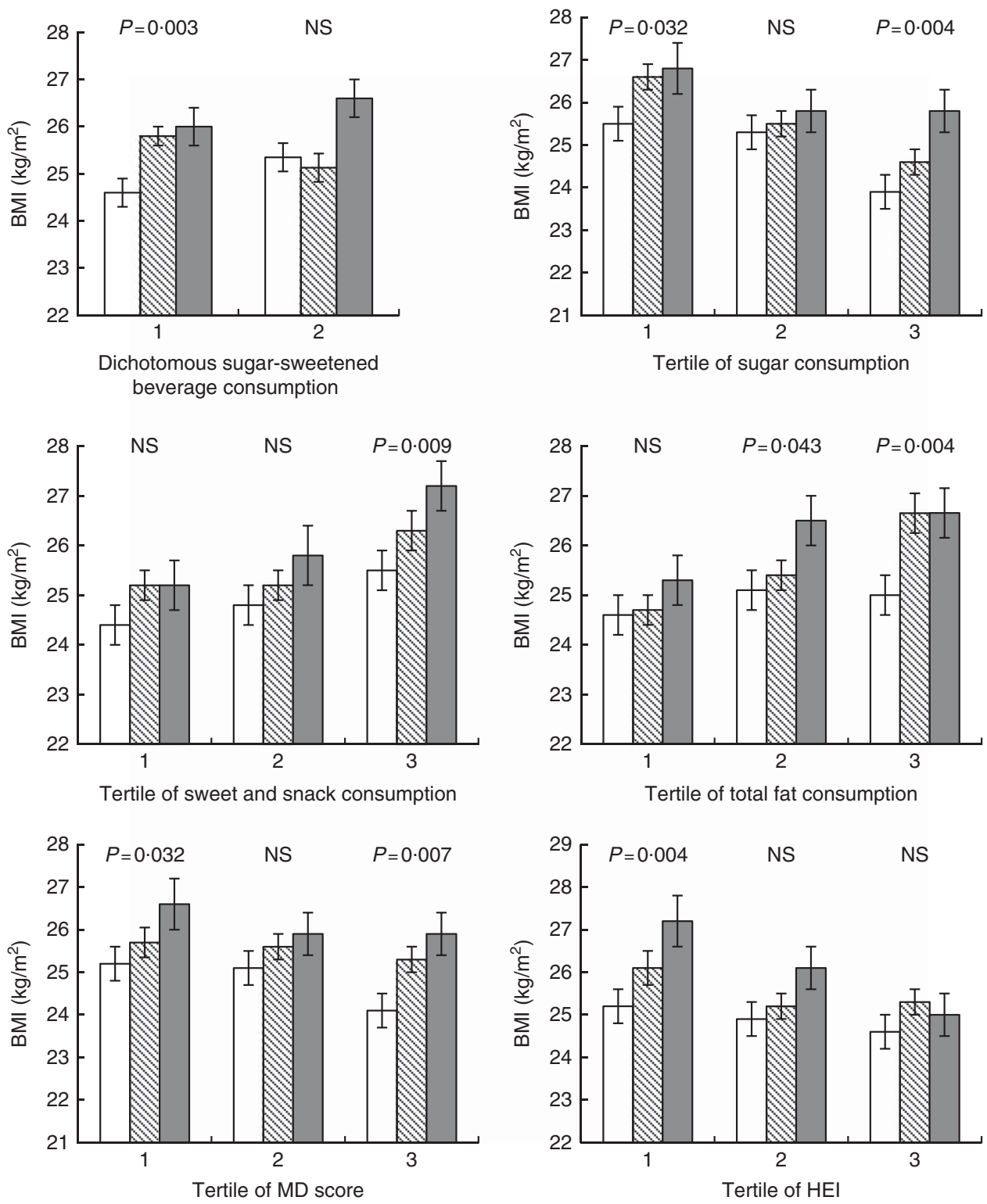

Fig 2. BMI by tertile or dichotomous food intake and tertile of dietary score and fat mass- and obesity-associated risk allele. Values represent least squares means, with their standard errors adjusted for age, sex, country, physical activity and smoking status; sugar-sweetened beverages: (1) $0 \mathrm{~g} / \mathrm{d}$, (2) $6.8-2191.5 \mathrm{~g} / \mathrm{d}$; sweets and snacks: (1) 0-55.1 g/d, (2) 55.3-105.6 g/d, (3) $105.8-693.9 \mathrm{~g} / \mathrm{d}$; percentage energy from sugars: (1) $5.1-18.3 \%$, (2) $18.3-23.0 \%$, (3) $23.0-47.9 \%$; percentage energy from fat: (1) 13.8-33.5\%, (2) 33.5-38.3\%, (3) 38.3-66.6\%; PREDIMED-based Mediterranean diet score (MD) (24): 3, high score (6-10), 2, intermediate score $(4-6), 1$, low score (0-4); Healthy Eating Index (HEI) ${ }^{(23)}: 3$, high index (11-46), 2, intermediate index (46-54), 1, low index (54-77). Results were deemed significant at $P<0.05$. No significant interactions were observed. $\square, T T ; \mathbb{S}, T A ; \square, A A$.

meta-analysis, the role of EI in the relationship between FTO and BMI remains unclear ${ }^{(10)}$ and other measures of PA, such as whether participants met PA recommendations, may have been more informative but were not the focus of the present analysis.

Although there have been a number of investigations of interactions between macronutrients intake, genotype and anthropometric measures, Qi et $a l .{ }^{(15)}$ were one of the first to evaluate relationships between specific food groups, genetic risk and adiposity. Some diet-gene interactions have been supported by findings from $\mathrm{RCT}^{(31-34)}$; however, few large-scale studies have identified any significant interaction between macronutrient intake and FTO genotype on $\mathrm{BMI}^{(12,35-40)}$.
A recent meta-analysis did not detect any interactions between protein intake and genetic predisposition to obesity on BMI, WC or waist:hip ratio ${ }^{(41)}$. In contrast, a very recent study reported a significant interaction between genetic risk score (based on sixteen obesity-related SNP) and intakes of energy, protein, total fat, SFA, PUFA and carbohydrate on BMI, body fat mass and $\mathrm{WC}^{(13)}$. The inconsistency of current evidence for interactions between macronutrients, FTO and adiposity, as well as the limited research into effects of specific food groups, highlights the need for further research in this area.

Our results support the need for personalising nutritional advice based on the rationale that diet and lifestyle behaviour 
responses will be bigger when individuals are given more accurate and relevant diagnostic feedback ${ }^{(42)}$. Focusing on health risks in the provision of lifestyle-based recommendations may facilitate long-term improvements in diet and $\mathrm{PA}^{(4,15,16)}$.

\section{Potential mechanisms}

Little is known about the mechanisms through which the FTO allele enhances obesity $\operatorname{risk}^{(6,43)}$, although recent evidence suggests that manipulation of a pathway for adipocyte thermogenesis regulation may play an important role ${ }^{(44,45)}$. In addition, there is little mechanistic explanation for the reported interaction between specific dietary components, FTO genotype and adiposity ${ }^{(15)}$. Intake of specific foods may be a marker for a less healthy diet and lifestyle ${ }^{(11)}$, and thus apparent relationships may not be causal.

The unhealthy food groups chosen for the present analyses were typically energy-dense foods, with limited nutritional value, low in fibre and with low satiety index ${ }^{(46)}$ but were highly palatable ${ }^{(47)}$. It has been speculated that the more attractive organoleptic properties generating through the frying process may drive associations between fried foods and increased risk of obesity ${ }^{(11)}$. These attributes may encourage higher ad libitum energy intake, and thereby mediate their effects on obesity. Nonetheless, our analyses were adjusted for EI, suggesting that the mechanism of action on adiposity goes beyond higher energy intake alone. To understand the mechanism(s) driving sustained surplus energy intake, it will be important to investigate simultaneous effects on energy expenditure.

\section{Strengths and limitations}

Strengths of our study include the relatively large number of European adults (1280), broadly representative of the seven countries in the Food4Me study in terms of diet and PA levels. We assessed dietary intakes using a semi-quantitative FFQ, enabling a more detailed appraisal of food groups and individual foods. Furthermore, we included an analysis of overall dietary healthfulness based on the MD score and on the HEI, which improved the richness of our dietary data. In addition, we evaluated the effect of diet-gene interactions on two measures of adiposity, BMI and WC, which have different interpretations and links with health outcomes.

A limitation of our study was that it was not powered to detect diet-gene interactions. In contrast, Qi et $a l^{(15)}$ included a much larger sample size of $n 37423$, which is likely to have contributed to the statistically significant interactions observed. In addition, we investigated the effect of only one obesity-related gene, although FTO is the gene with the largest association with adiposity, and the study would have been stronger if we had been able to use a risk score based on multiple gene variants ${ }^{(48)}$. Although Qi et al. ${ }^{(15)}$ studied effects of thirty-two SNP, the authors concluded that FTO was primarily responsible for the genetic associations observed. All FFQ-derived food intake data are subject to dietary misreporting ${ }^{(49)}$, although we did not identify any differences in dietary misreporting between FTO genotypes. As a sensitivity analysis, effects of over- and under-reporting of dietary intakes were minimised by excluding individuals with implausible energy intakes, and this exclusion did not alter the findings. Finally, anthropometric measures were self-measured and self-reported, which may introduce measurement errors. However, a validation study embedded within the Food4Me study demonstrated a high degree of correlation between self-reported and measured anthropometric variables (interclass correlation coefficients: height 0.990; weight 0.994; and BMI 0.983) ${ }^{(26)}$.

\section{Conclusions}

The present study has demonstrated that high consumption of some, but not all, unhealthy foods and the presence of poor dietary patterns in individuals with the FTO risk genotype are associated with greater BMI compared with individuals with no risk alleles. However, there was limited evidence of interactions between FTO genotype and dietary intakes on BMI. Research in larger cohorts is required to confirm or to refute these findings, and RCT will be needed to ascertain whether any associations are causal.

\section{Acknowledgements}

This work was supported by the European Commission under the Food, Agriculture, Fisheries and Biotechnology Theme of the 7 th Framework Programme for Research and Technological Development (265494).

The authors' contributions were as follows: Y. M., I. T., C. A. D., E. R. G., L. B., J. A. L., J. A. M., W. H. S., H. D., M. G. and J. C. M. contributed to the research design. J. C. M. was the Food4Me study leader. C. C.-M., C. F. M. M., H. F., C. B. O., C. W., A. L. M., R. F., S. N.-C., R. S.-C., S. K., L. T., C. P. L., M. G., A. S., M. C. W., E. R. G., L. B. and J. C. M. contributed to developing the standardised operating procedure for the study. C. C.-M., S. N.-C., R. S.-C., C. W., C. B. O., H. F., C. F. M. M., A. L. M., R. F., S. K., L. T., C. P. L., M. G., A. S., M. C. W. and J. C. M. conducted the intervention. C. C.-M., C. F. M. M. and W. H. S. contributed to physical activity measurements. K. M. L. and C. C.-M. wrote the paper and performed the statistical analysis for the manuscript and are joint first authors. All the authors contributed to a critical review of the manuscript during the writing process. All the authors approved the final version to be published.

None of the authors has personal or financial conflicts of interest.

\section{Supplementary material}

For supplementary material/s referred to in this article, please visit http://dx.doi.org/doi:10.1017/S0007114515004675

\section{References}

1. Organisation for Economic Co-operation and Development (2012) Health at a Glance: Europe 2012. OECD Publishing. http://dx.doi.org/10.1787/9789264183896-en

2. Ng M, Fleming T, Robinson M, et al. (2014) Global, regional, and national prevalence of overweight and obesity in children and adults during 1980-2013: a systematic analysis for the Global Burden of Disease Study 2013. Lancet 384, 766-781. 
3. van Vliet-Ostaptchouk JV, Snieder H \& Lagou V (2012) Gene-lifestyle interactions in obesity. Curr Nutr Rep 1, 184-196.

4. Ahmad S, Rukh G, Varga TV, et al. (2013) Gene $\times$ physical activity interactions in obesity: combined analysis of 111,421 individuals of European ancestry. PLoS Genet 9, e1003607.

5. Frayling TM, Timpson NJ, Weedon MN, et al. (2007) A common variant in the FTO gene is associated with body mass index and predisposes to childhood and adult obesity. Science 316, 889-894.

6. Locke AE, Kahali B, Berndt SI, et al. (2015) Genetic studies of body mass index yield new insights for obesity biology. Nature 518, 197-206.

7. Shungin D, Winkler TW, Croteau-Chonka DC, et al. (2015) New genetic loci link adipose and insulin biology to body fat distribution. Nature 518, 187-196.

8. Speakman JR, Rance KA \& Johnstone AM (2008) Polymorphisms of the FTO gene are associated with variation in energy intake, but not energy expenditure. Obesity (Silver Spring) 16, 1961-1965.

9. Corella D, Ortega-Azorín C, Sorlí JV, et al. (2012) Statistical and biological gene-lifestyle interactions of MC4R and FTO with diet and physical activity on obesity: new effects on alcohol consumption. PLOS ONE 7, e52344.

10. Livingstone KM, Celis-Morales C, Lara J, et al. (2015) Associations between FTO genotype and total energy and macronutrient intake in adults: a systematic review and metaanalysis. Obes Rev 16, 666-678.

11. Guallar-Castillón P, Rodríguez-Artalejo F, Fornés NS, et al. (2007) Intake of fried foods is associated with obesity in the cohort of Spanish adults from the European prospective investigation into cancer and nutrition. Am J Clin Nutr 86, 198-205.

12. Sonestedt E, Roos C, Gullberg B, et al. (2009) Fat and carbohydrate intake modify the association between genetic variation in the FTO genotype and obesity. Am J Clin Nutr $\mathbf{9 0}$, 1418-1425.

13. Goni L, Cuervo M, Milagro FI, et al. (2015) A genetic risk tool for obesity predisposition assessment and personalized nutrition implementation based on macronutrient intake. Genes Nutr 10, 445.

14. Brunkwall L, Ericson U, Hellstrand S, et al. (2013) Genetic variation in the fat mass and obesity-associated gene (FTO) in association with food preferences in healthy adults. Food Nutr Res 57, 10.3402/fnr.v57i0.20028.

15. Qi Q, Chu AY, Kang JH, et al. (2014) Fried food consumption, genetic risk, and body mass index: gene-diet interaction analysis in three US cohort studies. BMJ 348, 1610.

16. Qi Q, Chu AY, Kang JH, et al. (2012) Sugar-sweetened beverages and genetic risk of obesity. $N$ Engl J Med 367, 1387-1396.

17. Razquin C, Martinez JA, Martinez-Gonzalez MA, et al. (2010) A 3-year intervention with a Mediterranean diet modified the association between the rs9939609 gene variant in FTO and body weight changes. Int J Obes (Lond) 34, 266-272.

18. Celis-Morales C, Livingstone KM, Marsaux CFM, et al. (2015) Design and baseline characteristics of the Food4Me study: a web-based randomised controlled trial of personalised nutrition in seven European countries. Genes Nutr 10, 450

19. Livingstone K, Celis-Morales C, Navas-Carretero S, et al. (2015) Profile of European adults interested in internet-based personalised nutrition: the Food4Me study. Eur J Nutr (epublication 17 April 2015).

20. Forster H, Fallaize R, Gallagher C, et al. (2014) Online dietary intake estimation: the Food4Me food frequency questionnaire. J Med Internet Res 16, e150.
21. Fallaize R, Forster H, Macready LA, et al. (2014) Online dietary intake estimation: reproducibility and validity of the Food4Me food frequency questionnaire against a 4-day weighed food record. I Med Internet Res 16, e190.

22. Food Standards Agency (2002) McCance and Widdowson's The Composition of Foods, 6th ed. Cambridge: Royal Society of Chemistry.

23. Guenther PM, Casavale KO, Reedy J, et al. (2013) Update of the Healthy Eating Index: HEI-2010. I Acad Nutr Diet 113 , 569-580.

24. Estruch R, Ros E, Salas-Salvadó J, et al. (2013) Primary prevention of cardiovascular disease with a Mediterranean diet. $N$ Engl J Med 368, 1279-1290.

25. Martínez-González MÁ, Corella D, Salas-Salvadó J, et al. (2012) Cohort profile: design and methods of the PREDIMED study. Int J Epidemiol 41, 377-385.

26. Celis-Morales C, Livingstone KM, Woolhead C, et al. (2015) How reliable is Internet-based self-reported identity, sociodemographic and obesity measures in European adults? Genes Nutr 10, 476

27. Goldberg GR, Black AE, Jebb SA, et al. (1991) Critical evaluation of energy intake data using fundamental principles of energy physiology: 1 . Derivation of cut-off limits to identify under-recording. Eur J Clin Nutr 45, 569-581.

28. Henry CJK (2005) Basal metabolic rate studies in humans: measurement and development of new equations. Public Health Nutr 8, 1133-1152.

29. Hébert JR, Peterson KE, Hurley TG, et al. (2001) The effect of social desirability trait on self-reported dietary measures among multi-ethnic female health center employees. Ann Epidemiol 11, 417-427.

30. Schröder H, Fito M \& Covas MI (2007) Association of fast food consumption with energy intake, diet quality, body mass index and the risk of obesity in a representative Mediterranean population. Br J Nutr 98, 1274-1280.

31. Fisher E, Meidtner K, Ängquist L, et al. (2012) Influence of dietary protein intake and glycemic index on the association between TCF7L2 HapA and weight gain. Am J Clin Nutr $\mathbf{9 5}$ 1468-1476.

32. Qi Q, Bray GA, Smith SR, et al. (2011) Insulin receptor substrate 1 gene variation modifies insulin resistance response to weight-loss diets in a 2-year randomized trial: the Preventing Overweight Using Novel Dietary Strategies (POUNDS LOST) trial. Circulation 124, 563-571.

33. Xu M, Qi Q, Liang J, et al. (2013) Genetic determinant for amino acid metabolites and changes in body weight and insulin resistance in response to weight-loss diets: the Preventing Overweight Using Novel Dietary Strategies (POUNDS LOST) trial. Circulation 127, 1283-1289.

34. Mattei J, Qi Q, Hu FB, et al. (2012) TCF7L2 genetic variants modulate the effect of dietary fat intake on changes in body composition during a weight-loss intervention. Am J Clin Nutr 96, 1129-1136.

35. Grau K, Hansen T, Holst C, et al. (2009) Macronutrientspecific effect of FTO rs9939609 in response to a 10-week randomized hypo-energetic diet among obese Europeans. Int J Obes (Lond) 33, 1227-1234.

36. Moleres A, Ochoa MC, Rendo-Urteaga T, et al. (2012) Dietary fatty acid distribution modifies obesity risk linked to the rs9939609 polymorphism of the fat mass and obesityassociated gene in a Spanish case-control study of children. Br J Nutr 107, 533-538.

37. Lappalainen T, Lindström J, Paananen J, et al. (2012) Association of the fat mass and obesity-associated (FTO) gene variant (rs9939609) with dietary intake in the Finnish Diabetes Prevention Study. Br J Nutr 108, 1859-1865. 
38. Corella D, Arnett DK, Tucker KL, et al. (2011) A high intake of saturated fatty acids strengthens the association between the fat mass and obesity-associated gene and BMI. J Nutr 141, 2219-2225.

39. Phillips CM, Kesse-Guyot E, McManus R, et al. (2012) High dietary saturated fat intake accentuates obesity risk associated with the fat mass and obesity-associated gene in adults. $J$ Nutr 142, 824-831.

40. Ortega-Azorin C, Sorli J, Asensio E, et al. (2012) Associations of the FTO rs9939609 and the MC4R rs17782313 polymorphisms with type 2 diabetes are modulated by diet, being higher when adherence to the Mediterranean diet pattern is low. Cardiovasc Diabetol 11, 137.

41. Ankarfeldt MZ, Larsen SC, Ängquist L, et al. (2014) Interaction between genetic predisposition to adiposity and dietary protein in relation to subsequent change in body weight and waist circumference. PLOS ONE 9, e110890.

42. Martinez JA, Navas-Carretero S, Saris WHM, et al. (2014) Personalized weight loss strategies - the role of macronutrient distribution. Nat Rev Endocrinol 10, 749-760.
43. Speakman JR (2015) The 'fat mass and obesity related' (FTO) gene: mechanisms of impact on obesity and energy balance. Curr Obes Rep 4, 73-91.

44. Claussnitzer M, Dankel SN, Kim KH, et al. (2015) FTO obesity variant circuitry and adipocyte browning in humans. $N$ Engl J Med 373, 895-907.

45. Rosen CJ \& Ingelfinger JR (2015) Unraveling the function of FTO variants. $N$ Engl J Med 373, 964-965.

46. Bludell JE, Lawton CL, Cotton JR, et al. (1996) Control of human appetite: implications for the intake of dietary fat. Annu Rev Nutr 16, 285-319.

47. Fillion L \& Henry CJK (1998) Nutrient losses and gains during frying: a review. Int J Food Sci Nutr 49, 157-168.

48. Wang T, Jia W \& Hu C (2015) Advancement in genetic variants conferring obesity susceptibility from genome-wide association studies. Front Med 9, 146-161.

49. Johansson L, Solvoll K, Bjørneboe GE, et al. (1998) Under- and overreporting of energy intake related to weight status and lifestyle in a nationwide sample. Am J Clin Nutr $\mathbf{6 8}$, $266-274$. 